\title{
EFECTO DE LAS CARACTERISTICAS DE HARINA DE MADERA EN LAS PROPIEDADES FÍSICO-MECÁNICAS Y TÉRMICAS DE POLIPROPILENO RECICLADO
}

\section{EFFECTS OF THE CHARACTERISTICS OF WOOD FLOUR ON THE PHYSICO-MECHANICAL AND THERMAL PROPERTIES OF RECYCLED POLYPROPYLENE}

\author{
Justo Lisperguer ${ }^{1,2, \infty}$, Ximena Bustos², Yanina Saravia², \\ Carolina Escobar ${ }^{3}$, Henry Venegas ${ }^{3}$
}

\begin{abstract}
RESUMEN
Polipropileno reciclado $(\mathrm{rPP})$ proveniente de rafia sintética de grandes sacos postconsumo, fueron reforzados con 50 y $60 \%$ de harina de madera de Pinus radiata de dos tamaños de partícula (60 y 100 mesh) a través de un proceso de mezclado en fundido, con y sin polipropileno maleado (MAPP), utilizado como agente acoplante. La procesabilidad de rPP y de compositos a base de madera-rPP fue estudiada en términos del torque del proceso de mezcla, al igual que las propiedades físicas, térmicas y mecánicas, y comparadas con polipropileno virgen (PP).

Los resultados mostraron que la resistencia a la tracción y a la flexión (MOR), módulos determinados en tracción y en flexión (MOE) y resistencia al impacto de rPP y de los compositos madera-rPP, no decrecen comparadas con PP virgen y en términos de los módulos de elasticidad (MOE) y resistencia al impacto son significativamente superiores. Las propiedades mecánicas de las mezclas mejoraron levemente con la presencia de MAPP pero el tamaño de partícula y el contenido de harina de madera (50 y 60\%), no tuvieron una clara influencia en estas propiedades.

Calorimetría diferencial de barrido (DSC) fue utilizada para estudiar las propiedades de cristalización y fusión de las muestras y los resultados fueron comparados con PP virgen. Los resultados indican que la harina de madera actúa como un agente de nucleación incrementando la velocidad de cristalización, disminuyendo el grado de cristalización y en consecuencia disminuyendo ligeramente el punto de fusión de los compositos madera-rPP.

El análisis termogravimétrico (TGA), mostró que la estabilidad térmica de rPP y de los compositos maderarPP fue ligeramente superior a la de PP virgen.

En general, los resultados de este estudio muestran que es factible obtener materiales compuestos de buenas propiedades térmicas y mecánicas utilizando rPP postconsumo reforzado con harina de madera, en proporciones de 50 o $60 \%$ de contenido.
\end{abstract}

Palabras claves: Harina de madera, polipropileno reciclado, propiedades térmicas, propiedades mecánicas.

\footnotetext{
' Departamento de Química, Facultad de Ciencias, Universidad del Bío-Bío. Concepción. Chile.

${ }^{2}$ Centro de Investigación de Polímeros Avanzados. CIPA. Conicyt/Regional R08C1002. Concepción. Chile.

${ }^{3}$ Departamento de Ingeniería en Maderas, Facultad de Ingeniería, Universidad del Bío-Bío. Concepción. Chile.

- Autor para correspondencia: jlisperg@ubiobio.cl

Recibido: 28.08.2012. Aceptado: 02.01.2013.
} 


\begin{abstract}
Recycled polypropylene (rPP) from synthetic raffia big bags was reinforced with 50 and $60 \%$ of Pinus radiata wood flour of two particle size (60 and $100 \mathrm{mesh}$ ) through a blending process with and without maleated polypropylene (MAPP), used as a coupling agent. The processability of the pure rPP and wood-rPP composites was studied in terms of the mixing process torque and also the physico-mechanical properties and then compared with a commercial virgin polypropylene (PP). The results showed that the tensile and flexural strength (MOR), tensile and flexural modulus (MOE) and impact strength of the pure and reinforced $\mathrm{rPP}$, did not decrease in comparison with the virgin PP, and in terms of the elasticity modulus (MOE) and impact strength, the rPP composites were superior to the virgin plastic. The mechanical properties were slightly enhanced by the MAPP, but the particle size and the filler content (50 and 60\%) had no clear influence on the mechanical properties.

Differential scanning calorimetry (DSC) was used to study the crystallization and melting behaviour of the rPP and rPP-wood composites and the results were compared with the virgin PP. The DSC analysis indicated that the wood flour particles acted as a nucleating agent, increasing crystallization rate and decreasing the crystallinity degree, and consequently, decreasing slightly the melting temperature of the $\mathrm{rPP} /$ wood composites.
\end{abstract}

Thermogravimetric analysis (TGA) revealed that the thermal stability of the $\mathrm{rPP}$ and $\mathrm{rPP} /$ wood composites were slightly greater than that of the virgin PP.

In general, the results of this study showed that it is feasible to obtain composite materials with good thermal and mechanical properties with recycled PP from postconsumer synthetic raffia reinforced with 50 or $60 \%$ wood flour.

Keywords: Wood flour, recycled polypropylene, mechanical properties, thermal properties.

\title{
INTRODUCCIÓN
}

El continuo incremento en la producción y consumo de varios materiales plásticos de corta vida útil, especialmente utilizados en empaques, ha producido un problema crítico en las últimas décadas en términos de volúmenes desechados en vertederos municipales, por problemas medioambientales, económicos, sociales y políticos.

Uno de los termoplásticos de mayor uso en el mundo, después del polietileno de alta densidad (HDPE) y polietileno de baja densidad (LDPE) es el polipropileno (PP) (Brachet et al. 2008).

Entre los diversos productos manufacturados basados en PP se encuentran empaques manufacturados con rafia sintética, bolsas y sacos en diversos formatos, envases para alimentos, etc.

El PP ha sido utilizado, desde hace algunos años en la elaboración de materiales plástico-madera, donde se refuerza con fibras vegetales o harina de madera para la manufactura de productos para la construcción como madera sintética y paneles para puertas, ventanas y cercos ( Salemane y Luyt 2006, Clemons y Caufield 2005).

Debido a la naturaleza no polar de plásticos como PP y la naturaleza polar de los componentes de la madera como celulosa, hemicelulosa y lignina, los materiales plástico-madera forman fases inmiscibles que deterioran las propiedades mecánicas y térmicas de estos materiales (Xiong et al. 2009, Keener et al. 2004, Dikove y Luyt 2010). Esto ha significado que gran parte de la investigación en este tema, se ha enfocado en modificar la madera, el polímero o usar agentes acoplantes para mejorar la interacción entre plástico y madera.

Borysiak y Doczekalska (2006), encontraron que la esterificación de la madera con anhídrido maleico incrementaba la cristalinidad de los materiales plástico-madera, la dureza y rigidez del material. 
Bouza et al. (2008), investigaron el efecto de viniltrimetoxi silano como agente acoplante para compositos madera-PP. Los resultados mostraron que el uso de hasta un 30\% de madera con pequeñas cantidades de agente acoplante tenía una conducta similar a la de un agente acoplante comercial como PP injertado con anhídrido maleico (MAPP).

Otros investigadores (Núñez et al. 2003, Keener et al. 2004), estudiaron la conducta mecánica de diferentes mezclas de harina de madera-PP, modificando la interfase madera-PP con un copolímero de MAPP, encontrando que pequeñas cantidades del agente acoplante mejoraba la dispersión de la madera en el polímero.

Otro trabajo utilizó fibras vegetales para reforzar mezclas de HDPE y PP (Selke y Wichman 2004). Ellos encontraron que un 30\% de fibra mejoraba significativamente el módulo de elasticidad de mezclas 30:70 (PP/PE).

La mayor parte de las investigaciones con PP se han concentrado en el uso de PP virgen, sólo o mezclado con otros termoplásticos.

Existen pocos trabajos sobre materiales PP-madera usando polipropileno reciclado (rPP)

En un trabajo reciente Ashori y Nourbakhsh (2009), estudiaron las propiedades físicas y mecánicas de paneles manufacturados por un método de prensado en caliente usando HDPE y rPP reforzados con fibra proveniente de papel de diarios. Los paneles basados en HDPE tenían mejores propiedades mecánicas que los paneles basados en rPP.

En otro trabajo Adhikary et al. (2010), investigaron los efectos de ciclos de congelamiento y descongelación acelerados en HDPE virgen y reciclado mezclado con PP y reforzado con dos composiciones diferentes de harina de madera. En general, los compositos basados en polímeros reciclados mostraron absorción e hinchamiento más bajos que polímeros vírgenes, pero sus propiedades mecánicas fueron ligeramente inferiores.

Spinacé et al. (2009), usaron fibras vegetales de Curaua (Ananas erectifolius) para reforzar PP reciclado a través de un proceso de extrusión. Encontraron que el módulo de Young de los compositos se incrementó con un contenido de hasta $40 \%$ de fibra, pero la resistencia al impacto alcanzó los mayores valores a un contenido de $20 \%$ de fibra.

Najafi et al. (2006) usaron harina de madera de abeto para reforzar HDPE y PP, tanto vírgenes como reciclados. Los compositos a base de PP mostraron mayor rigidez que los compositos a base de HDPE, pero menor resistencia al impacto.

Dányádi et al. (2010), compararon el efecto de MAPP, dos surfactantes (ácido esteárico y palmitato de celulosa) y la modificación química de la madera (bencilación), en la adhesión interfacial, homogenidad, procesabilidad y absorción de agua de compositos a base de PP y harina de madera. La bencilación produce una disminución de la adhesión interfacial mientras MAPP no tiene mayor influencia. La homogenidad, viscosidad y absorción de agua prácticamente no cambian y son independientes de la cantidad de agente acoplante usado. Los surfactantes mejoran claramente la homogenidad y procesabilidad del material.

Los trabajos existentes muestran que la harina de madera en algunos casos incrementa el módulo de elasticidad, pero en otros disminuye la resistencia a la tracción y al impacto debido a la contaminación de PP con otros termoplásticos cuando las tecnologías de recolección y selección del material no son bastante eficientes (Al-Salem et al. 2009).

El objetivo del presente trabajo se orientó a estudiar el efecto de harina de madera sobre rPP obtenido de sacos fabricados con rafia sintética, variando el tamaño de partículas de madera y utilizando dos cargas diferentes de madera en la matriz polimérica.

El PP postconsumo proveniente de rafia no está contaminado con otros materiales plásticos, se encuentra en grandes volúmenes en vertederos y puede ser seleccionado y recolectado eficientemente. 
El uso de rPP en materiales plástico-madera tiene ventajas económicas, energéticas y medioambientales respecto del uso de plásticos vírgenes.

Para mejorar la interacción entre harina de madera y rPP se utilizó un pequeño porcentaje de MAPP como agente acoplante y se estudiaron las propiedades mecánicas y térmicas de los compositos, comparándolas con las propiedades de compositos fabricados con PP virgen.

\section{MATERIALES Y MÉTODOS}

Materiales

Harina de madera de Pinus radiata fue obtenida de un aserradero industrial, secada durante $12 \mathrm{~h} \mathrm{a} 105^{\circ} \mathrm{C}$, tamizada a 60 y 100 mesh y secada nuevamente por $24 \mathrm{~h}$, hasta un contenido de humedad de 2-3-\%.

El PP virgen fue suministrado por PETROQUIM- Chile (PH 0320) con un índice de fluidez (MFI) de $3.3 \mathrm{~g} / 10$ min a $190^{\circ} \mathrm{C}$ y carga de $2.16 \mathrm{~kg}$, para ser utilizado como control.

La rafia sintética proveniente de sacos fue recolectada, limpiada y procesada a pellets de rPP por REPROCESA S.A. (industria chilena recicladora de plásticos). El MFI de rPP fue medido en un equipo DYNISCO LMI de acuerdo a la norma ASTM D1238-04 a $190^{\circ}$ y $2.16 \mathrm{~kg}$ de carga y fue de $5.1 \mathrm{~g} / 10 \mathrm{~min}$.

El agente acoplante utilizado fue polipropileno maleado (MAPP, Epolene E-43) y fue suministrado por Eastman Chemical Products, Inc.

\section{Proceso de mezclado en fundido}

La harina de madera de Pinus radiata fue utilizada en dos porcentajes diferentes para reforzar rPP: 50 y $60 \%$ en peso. Para cada formulación se utilizaron dos tamaños de partículas; 60 mesh $(0.250 \mathrm{~mm})$ y 100 mesh $(0.149 \mathrm{~mm})$. Se utilizó también $1 \%$ en peso de MAPP, para estudiar la influencia de este agente acoplante en las propiedades de los compositos.

La tabla 1 muestra las composiciones de las diferentes muestras usadas para el estudio.

Tabla 1. Formulación de los compositos de harina de madera y rPP.

\begin{tabular}{|c|c|c|c|c|c|}
\hline \multirow[t]{2}{*}{ Muestras } & \multirow[t]{2}{*}{$\begin{array}{c}\text { PP virgen } \\
(\%)\end{array}$} & \multirow[t]{2}{*}{$\begin{array}{l}\mathbf{r P P} \\
(\%)\end{array}$} & \multicolumn{2}{|c|}{$\begin{array}{c}\text { Harina de madera } \\
(\%)\end{array}$} & \multirow[t]{2}{*}{$\begin{array}{c}\text { MAPP } \\
(\%)\end{array}$} \\
\hline & & & 60 mesh & 100 mesh & \\
\hline vPP & 100 & - & - & - & - \\
\hline rPP & - & 100 & - & - & - \\
\hline $\mathrm{A}$ & - & 49 & 50 & - & 1 \\
\hline $\mathrm{B}$ & - & 50 & 50 & - & - \\
\hline $\mathrm{C}$ & - & 49 & - & 50 & 1 \\
\hline $\mathrm{D}$ & - & 50 & & 50 & - \\
\hline$E$ & - & 39 & 60 & - & 1 \\
\hline $\mathrm{F}$ & - & 40 & 60 & - & - \\
\hline $\mathrm{G}$ & - & 39 & - & 60 & 1 \\
\hline $\mathrm{H}$ & - & 40 & - & 60 & - \\
\hline
\end{tabular}


Las mezclas fueron preparadas en un equipo Thermo Haake Polydrive Rheomix 600, provisto de un rodillo mezclador de paletas y un medidor del torque, a una temperatura de $180^{\circ} \mathrm{C}$, velocidad rotacional de $50 \mathrm{rpm}$ durante 15 minutos.

Los componentes de las mezclas fueron agregados secuencialmente al mezclador. Primero se agregó rPP y MAPP y después de 5 minutos de mezcla se agregó la harina de madera y se mantuvo el proceso durante 10 minutos más.

Las mezclas extruídas fueron posteriormente moldeadas en una prensa de laboratorio, a $190^{\circ} \mathrm{C}$ y 100 bar de presión para obtener las probetas para los ensayos de tracción, flexión, impacto y para las propiedades térmicas de las mezclas.

\section{Ensayos físicos y mecánicos}

Las propiedades físicas de los compositos de madera-rPP fueron ensayados para determinar la absorción de agua e hinchamiento, de acuerdo a la norma ASTM D 1037-96a. Se evaluaron 10 probetas de $3 \times 50$ x $50 \mathrm{~mm}^{3}$ después de ser sumergidas en agua a $20 \pm 3^{\circ} \mathrm{C}$ por 24 horas. También se determinó la gravedad específica y el perfil de densidad de los compositos, mediante un perfilómetro Amersham, modelo AMCK 6693 provisto de una pastilla de Am-241.

Los ensayos de tracción y flexión de PP virgen, rPP y compositos madera-rPP se realizaron con una máquina de ensayos universal Instron 4468 provista de una celda de carga de $5000 \mathrm{~kg}$ de capacidad. La velocidad para los ensayos de tracción y flexión fue de $5 \mathrm{~mm} / \mathrm{min}$, de acuerdo a la norma ASTM D $638-03$ y de $1.28 \mathrm{~mm} / \mathrm{min}$ de acuerdo a la norma ASTM D 790 - 03, respectivamente. Todos los ensayos fueron realizados a temperatura ambiente de $23^{\circ} \mathrm{C}$ y $60 \%$ de humedad relativa. Se determinaron los promedios y desviaciones estándar de 10 probetas de cada formulación.

El ensayo de resistencia al impacto Izod fue realizado a temperatura ambiente de acuerdo a la norma ASTM D 256 - 03 utilizando 10 probetas con entalle para cada formulación en una máquina de péndulo Dynisco - API.

Se efectuó un análisis paramétrico de varianza a los datos recolectados para distinguir las diferencias estadísticas $(\mathrm{p} \leq 0.05)$ entre las propiedades de la muestra. Todos los análisis estadísticos fueron realizados con el software Statgraphics Plus.

\section{Ensayos de DSC y TGA}

Estudios de cristalización y fusión de los compositos y de PP y rPP se realizaron utilizando un equipo Mettler Toledo DSC $822^{\mathrm{e}}$ provisto de un software $\mathrm{STAR}^{\mathrm{e}}$ para el análisis de los datos.

Se ensayaron muestras de $5-10 \mathrm{mg}$ a una velocidad de calentamiento de $10^{\circ} \mathrm{C} / \mathrm{min}$ en una atmósfera de nitrógeno, entre 30 y $200^{\circ} \mathrm{C}$. De los termogramas se obtuvo la temperatura de fusión $\left(\mathrm{T}_{\mathrm{m}}\right)$ y la entalpía de fusión $\left(\Delta \mathrm{H}_{\mathrm{m}}\right)$ del cambio desde el sólido cristalino al polímero amorfo fundido.

La temperatura de cristalización $\left(\mathrm{T}_{\mathrm{c}}\right)$ y el grado de cristalinidad de las muestras $\left(\mathrm{X}_{\mathrm{c}}\right)$ fue obtenido del cambio exotérmico producido al enfriar una muestra fundida desde $200^{\circ} \mathrm{C}$ hasta temperatura ambiente, a una velocidad de enfriamiento de $10^{\circ} \mathrm{C} / \mathrm{min}$, bajo una atmósfera de nitrógeno. El grado de cristalinidad fue obtenido de esta exoterma, considerando un valor $209 \mathrm{~J} / \mathrm{g}$ para el PP 100\% cristalino (Galeski 1999).

Se estudió la estabilidad de PP (virgen y reciclado) y de los compositos madera-rPP, a través de ensayos no isotérmicos, utilizando un analizador termogravimétrico TGA Q 50 de TA Instruments.

Los ensayos fueron realizados a una velocidad de calentamiento de $10^{\circ} \mathrm{C} /$ min con muestras de masa promedio de $6 \mathrm{mg}$, bajo una atmósfera de nitrógeno, en un rango de temperatura entre 25 y $600^{\circ} \mathrm{C}$. 


\section{RESULTADOS Y DISCUSIÓN}

\section{Propiedades físicas}

En la tabla 2 se muestran los resultados de hinchamiento, absorción de agua y densidad promedio de los compositos madera-rPP con y sin agente acoplante MAPP. Los valores de absorción e hinchamiento corresponden al promedio de 10 réplicas para cada muestra.

Tabla 2. Propiedades físicas de los compositos.

\begin{tabular}{|c|c|c|c|}
\hline Muestras & $\begin{array}{c}\text { Gravedad } \\
\text { específica } \\
\left(\mathbf{k g} / \mathbf{m}^{\mathbf{3}}\right)\end{array}$ & $\begin{array}{c}\mathbf{2 4 - h} \\
\text { Absorción (\%) }\end{array}$ & $\begin{array}{c}\mathbf{2 4 - h} \\
\text { Hinchamiento } \\
\mathbf{( \% )}\end{array}$ \\
\hline vPP & 923 & $0.00(0.00)$ & $0.93(0.00)$ \\
\hline rPP & 1006 & $0.00(0.00)$ & $2.96(0.00)$ \\
\hline A & 1122 & $1.29(0.21)$ & $7.73(3.19)$ \\
\hline B & 1094 & $1.50(0.28)$ & $8.77(0.06)$ \\
\hline C & 1108 & $1.47(0.14)$ & $9.60(1.96)$ \\
\hline D & 1100 & $1.59(0.05)$ & $8.17(2.53)$ \\
\hline E & 1051 & $1.92(0.27)$ & $9.12(0.54)$ \\
\hline F & 1138 & $2.63(0.23)$ & $10.20(1.48)$ \\
\hline G & 1198 & $2.00(0.10)$ & $8.99(1.38)$ \\
\hline H & 1102 & $2.94(0.02)$ & $8.12(1.10)$ \\
\hline
\end{tabular}

( ) Los valores dados entre paréntesis corresponden a la desviación estándar.

Como se aprecia en la tabla 2, es razonable esperar un aumento de la absorción de agua e hinchamiento cuando aumenta el porcentaje de harina de madera de $50 \%$ (A, B, C, D) a $60 \%$ (E, F, G, H) en la matriz polimérica. Para los compositos a base de rPP, los valores del espesor por hinchamiento variaron desde 7.73 a $10.20 \%$. El agente acoplante no tuvo influencia en esta propiedad de los compositos y las diferencias entre compositos con 60 y 100 mesh de tamaño de partícula, no fueron significativas.

En otros estudios (Dányádi et al. 2010) la adición de MAPP mejora la adhesión interfacial entre madera y la matriz polimérica pero no altera la absorción de agua de los compositos. En otro trabajo (Ye et al. 2013), la presencia de MAPP mejora la resistencia a la tracción e incrementa notablemente la resistencia al impacto.

El incremento de la adhesión interfacial con la presencia de MAPP parece influir en forma más directa en las propiedades mecánicas pero no es suficiente para afectar la absorción de agua e hinchamiento.

\section{Estudio del torque del proceso de mezcla}

Se estudió la influencia que tuvo la harina de madera en el torque del proceso de fundido y extrusión del rPP y se comparó con PP virgen. La figura 1 muestra los resultados del torque medido en el proceso de mezcla de rPP con 50\% de harina de madera, con tamaños de partícula de 60 y 100 mesh, con MAPP y sin MAPP. Se comparan, además, con los valores de torque de rPP y PP virgen puros. 


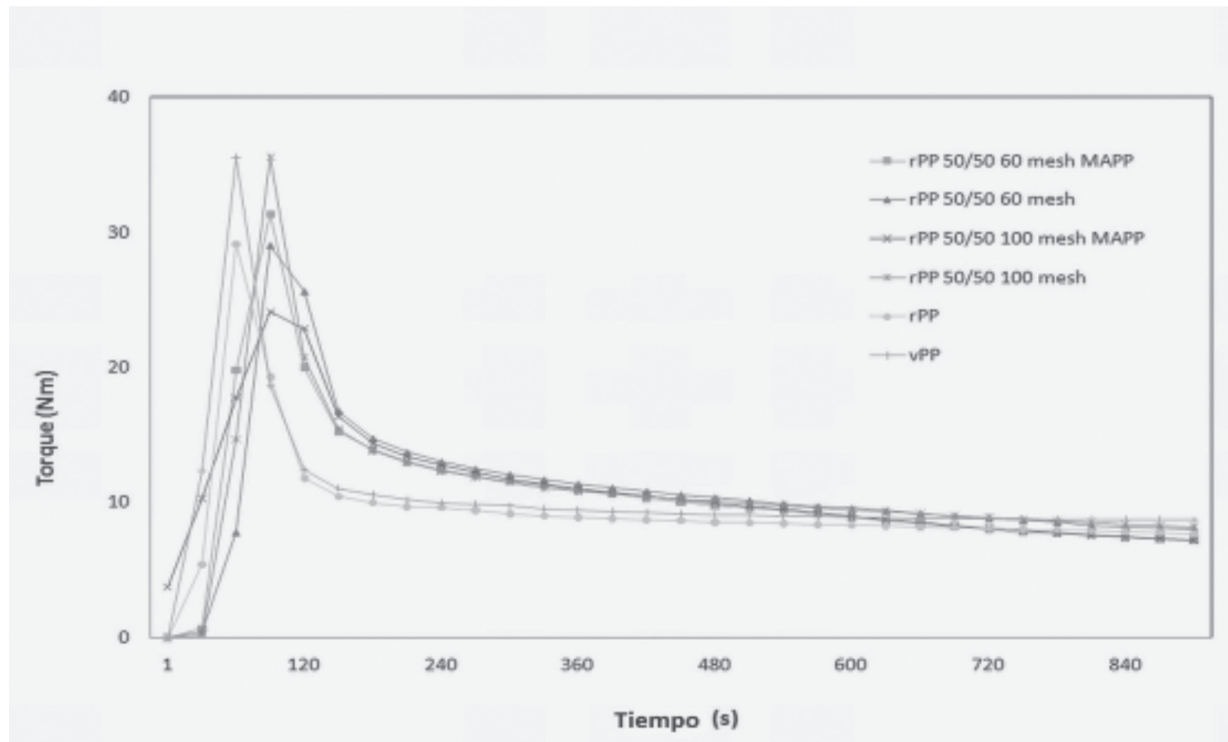

Figura 1. Curvas del torque de polímeros puros y de compositos de rPP con 50\% de madera.

En la figura 1 se aprecia un fuerte incremento en los valores del torque cuando se incorpora harina de madera en el mezclador, después de 5 minutos de la adición de rPP y el compatibilizador. Al final del proceso de mezcla, el torque del PP virgen, rPP y de los compositos madera-rPP, se estabilizan a un valor similar de 9 Nm aproximadamente. Los valores de torque para los compositos con MAPP disminuyen ligeramente bajo $9 \mathrm{Nm}$ sin mayores diferencias para los tamaños de partículas de 60 y 100 mesh. La figura 2 muestra los resultados del torque medido para las mezclas de rPP con 60\% de harina de madera a un tamaño de partículas de 60 y 100 mesh con y sin MAPP.

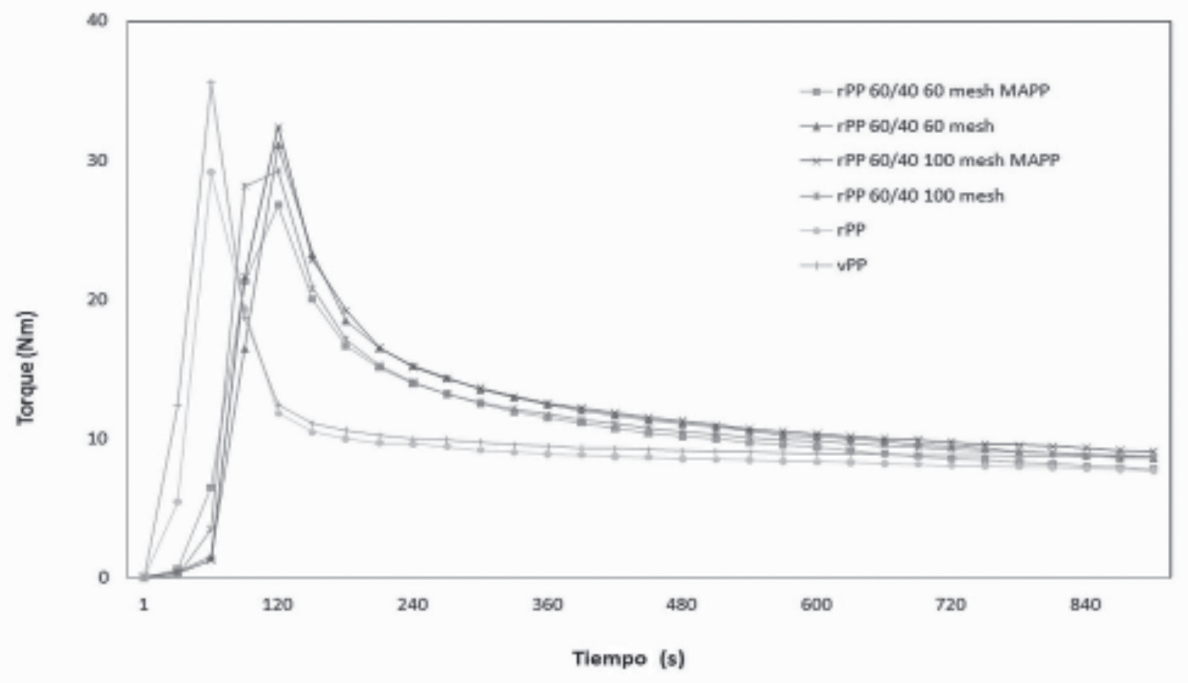

Figura 2. Curvas del torque de polímeros puros y de compositos de rPP con $60 \%$ de madera. 
La adición de $60 \%$ de harina de madera a los compositos no cambia significativamente los valores del torque de las diferentes muestras. El torque de estos compositos se estabilizó a valores de $10 \mathrm{Nm}$ aproximadamente.

En general, las figuras 1 y 2 presentan conductas similares en términos del torque de PP virgen, rPP y compositos rPP-madera. Tampoco se aprecia en las figuras diferencias de los valores del torque entre compositos con madera de 60 mesh y 100 mesh.

\section{Propiedades mecánicas}

La figura 3 muestra los resultados de la resistencia a la tracción (MOR) de PP virgen, rPP y compositos maderarPP.

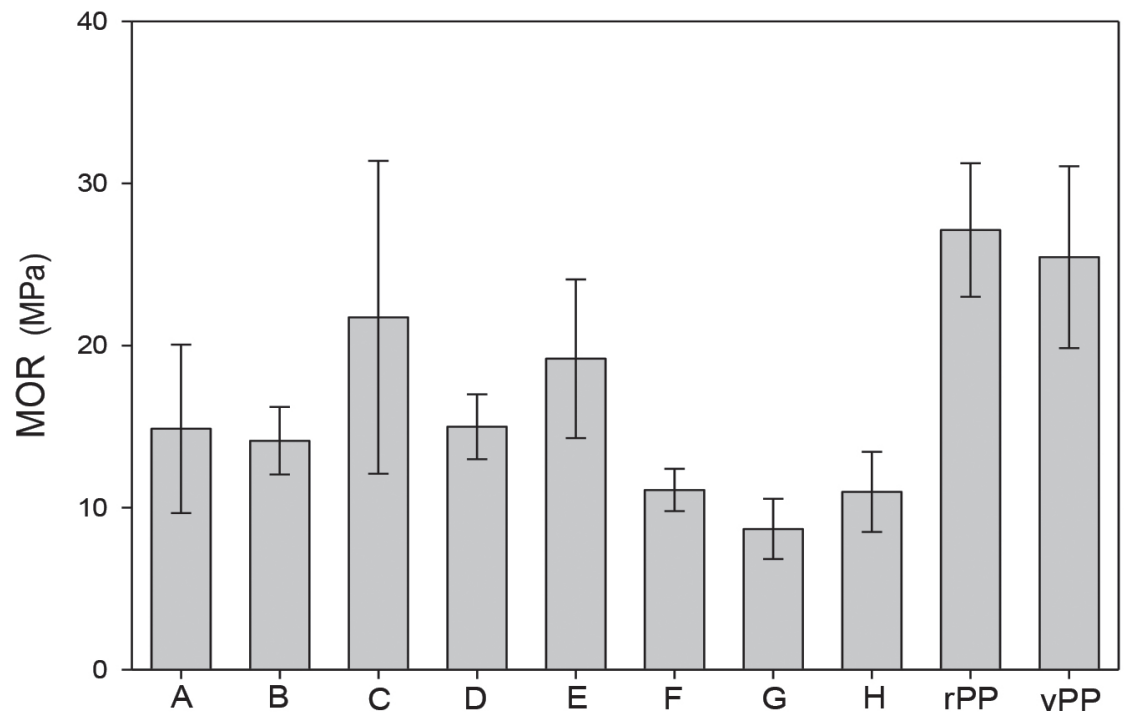

Figura 3. MOR de polímeros puros y de compositos reforzados con $50 \%$ y $60 \%$ de harina de madera determinado en ensayos de tracción.

Como se aprecia en la figura 3, el rPP mostró el valor más alto, ligeramente superior a PP virgen. Cuando se incorpora $50 \%$ de harina de madera a rPP la resistencia a la tracción disminuye para los dos tamaños de partícula de 60 y 100 mesh. La presencia del compatibilizador, MAPP, incrementa ligeramente la resistencia a la tracción (C).

La adición de $60 \%$ de harina de madera produjo la mayor disminución de la resistencia a la tracción de los compositos, a valores bajo $10 \mathrm{MPa}$, especialmente para compositos con madera de 100 mesh $(\mathrm{G}, \mathrm{H})$.

Publicaciones recientes (Haque et al. 2009), han reportado que 15\% de fibras de coco de palma utilizados como refuerzo de PP virgen disminuían ligeramente la resistencia a la tracción. Cargas de fibra superiores a este valor disminuían fuertemente los valores de la resistencia a la tracción. 
La figura 4 muestra los valores del módulo de tracción (MOE) de PP virgen, rPP y compositos de madera-rPP.

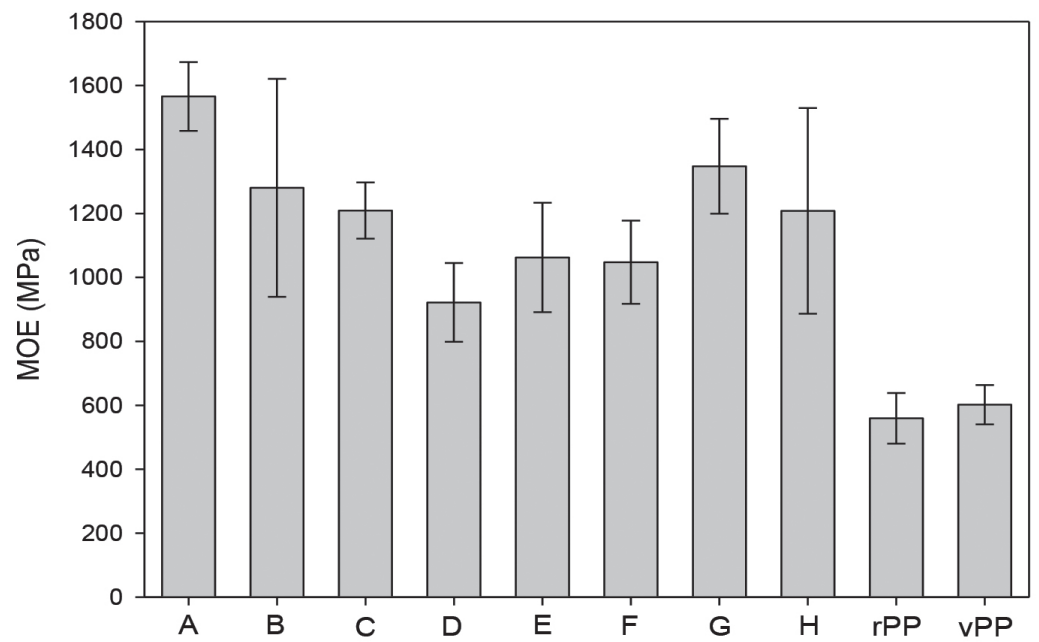

Figura 4. MOE de polímeros puros y de compositos reforzados con $50 \%$ y $60 \%$ de harina de madera determinado en ensayos de tracción.

En general, es posible apreciar un claro incremento en el módulo de los compositos, comparado con los polímeros puros. El mayor incremento lo experimentan las mezclas que contienen el compatibilizador MAPP, para las dos composiciones de madera y los dos tamaños de partículas.

El análisis estadístico mostró que no había diferencias significativas entre compositos que contenían 50 y $60 \%$ de harina de madera. El análisis también reveló que no existe una clara tendencia en la influencia del tamaño de partícula en el módulo de tracción de los compositos.

La figura 5 muestra la resistencia a la flexión (MOR) de PP virgen, rPP y compositos de madera-rPP.

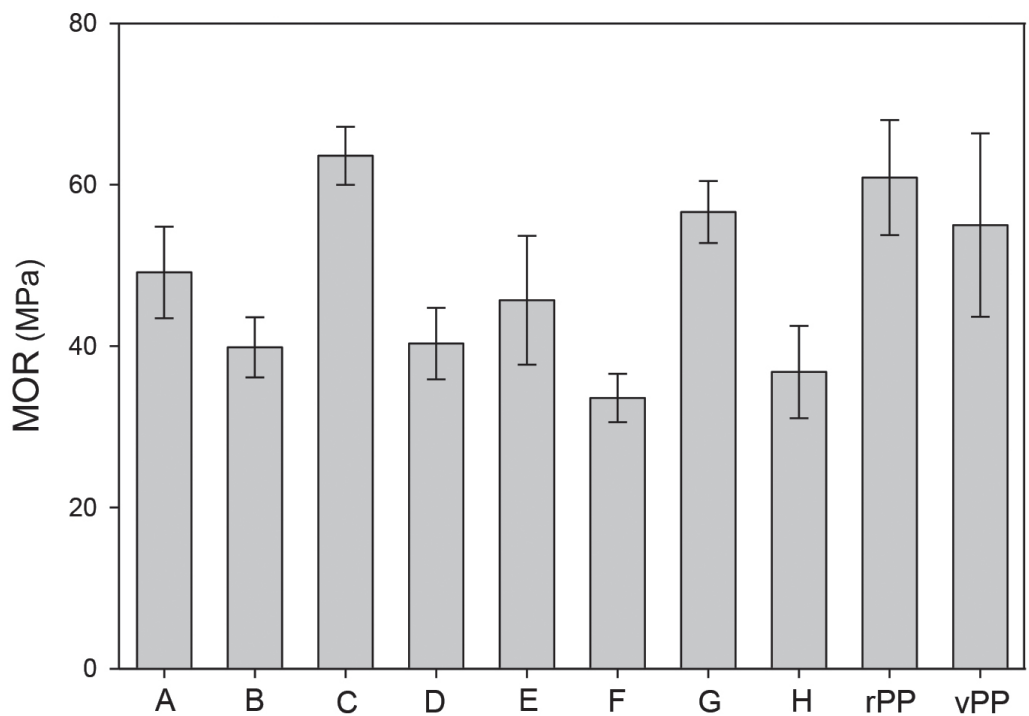

Figura 5. MOR de polímeros puros y de compositos reforzados con 50 y $60 \%$ de harina de madera, determinados en ensayos de flexión. 
En la figura se aprecia que rPP tiene valores de resistencia a la flexión ligeramente superiores a PP virgen. La incorporación de 50\% de harina de madera de 100 mesh con MAPP(C), incrementa ligeramente el MOR sobre rPP. Para los otros compositos con 60\% de harina de madera (60 y 100 mesh), los valores decrecen respecto de PP y rPP.

Por otra parte, se aprecia claramente en la figura 5 que los compositos que contienen MAPP presentan valores del MOR superiores a los compositos que no contienen agente acoplante.

Los valores del MOE, determinados en ensayos de flexión, de los polímeros y de los compositos se muestran en la figura 6.

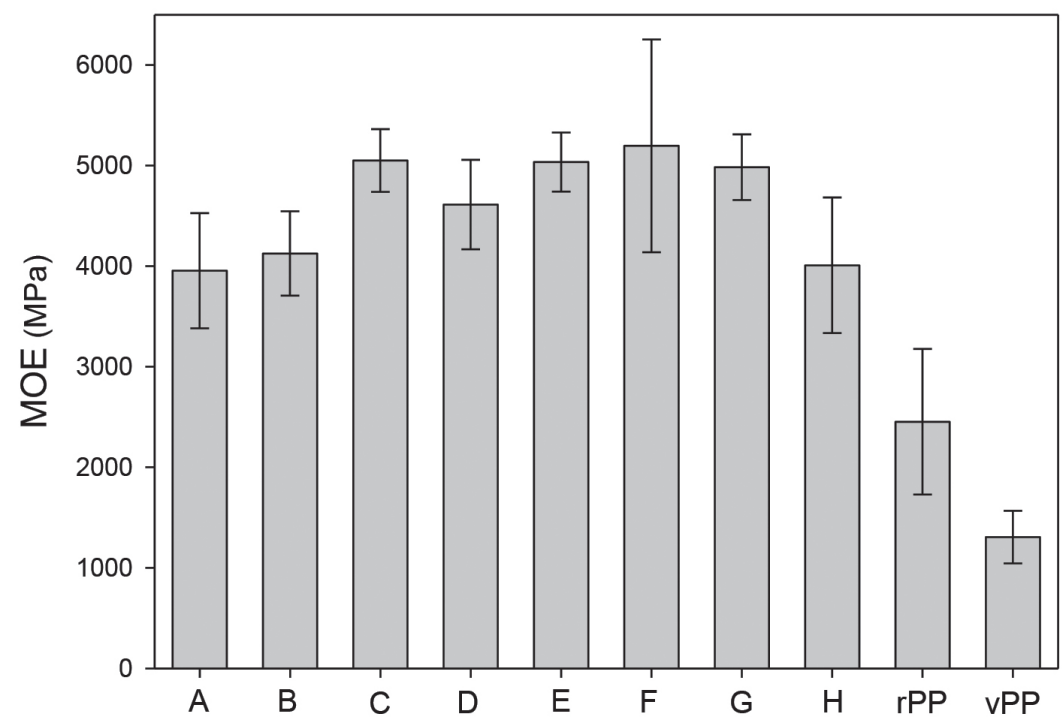

Figura 6. MOE de polímeros puros y de compositos con 50 y $60 \%$ de harina de madera, determinados en ensayos de flexión.

Como se muestra en la figura 6, la incorporación de harina de madera incrementa fuertemente los valores del MOE de los compositos. El mayor incremento se produjo en compositos que contenían $60 \%$ de harina de madera. El tamaño de partícula no muestra una influencia definida en los valores del MOE de los compositos.

La presencia de madera en la matriz polimérica de rPP incrementa la rigidez del polímero, incrementando fuertemente los valores del MOE, respecto de los polímeros puros. Este fenómeno ha sido también reportado para otros polímeros por investigadores que estudiaron el efecto de harina de madera en mezclas de PS reciclado y PE (Simonsen y Rials 1996, Choi et al. 2006, Lisperguer et al. 2011).

Spinacé et al. (2009), informan incrementos de 51\% en la resistencia y módulo de flexión de PP reforzado con $20 \%$ de fibras de Curaua y un incremento de un $30 \%$ en las propiedades de tracción de los mismos compositos. 
La figura 7 muestra la resistencia al impacto Izod con muestras con entalle de los polímeros puros y de los compositos reforzados con harina de madera.

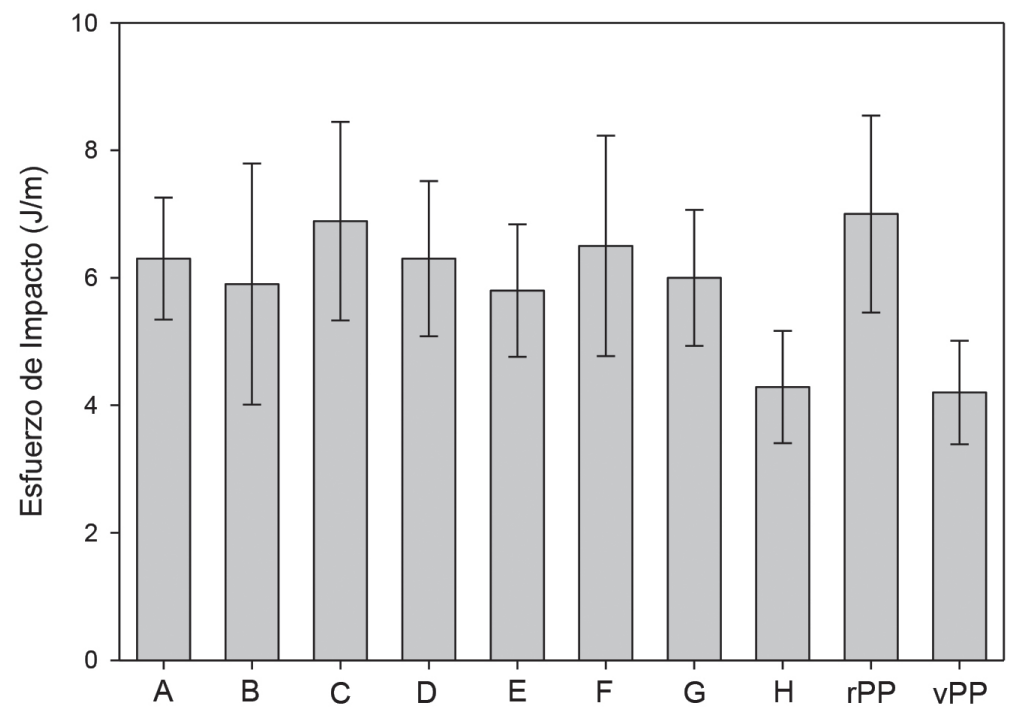

Figura 7. Resistencia al impacto de polímeros puros y de compositos reforzados con 50 y $60 \%$ de harina de madera.

Como se aprecia en la figura 7 los valores de resistencia al impacto de rPP y de los compositos madera-rPP se incrementan fuertemente, comparados con PP virgen. En general, los resultados de la medición de las propiedades mecánicas como el MOE en tracción, MOR y MOE en flexión y resistencia al impacto Izod de los compositos de rPP reforzados con harina de madera de 60 y 100 mesh, muestran valores superiores al PP virgen. Los compositos que contienen MAPP, muestran valores ligeramente superiores a los compositos sin este compuesto, lo que muestra un efecto compatibilizador positivo para las mezclas madera-rPP debido probablemente a un ligero incremento de adhesión interfacial.

\section{Análisis térmico}

El análisis DSC fue utilizado para estudiar el comportamiento de la fusión y los cambios en la cristalinidad de rPP, al incorporar harina de madera. Estas propiedades fueron comparadas con PP virgen. La tabla 3 muestra los puntos de fusión $\left(\mathrm{T}_{\mathrm{m}}\right)$, entalpías de fusión $\left(\Delta \mathrm{H}_{\mathrm{m}}\right)$, temperatura media de cristalización $\left(\mathrm{T}_{\mathrm{c}}\right)$ y porcentaje de cristalinidad $\left(\mathrm{X}_{\mathrm{c}}\right)$ de las muestras. 
Tabla 3. Propiedades de fusión y cristalización de vPP, rPP y compositos harina de madera-rPP.

\begin{tabular}{|c|l|l|l|l|}
\hline Muestras & $\mathbf{T}_{\mathbf{m}}\left({ }^{\mathbf{0}} \mathbf{C}\right)$ & $\mathbf{\Delta} \mathbf{H}_{\mathbf{m}}(\mathbf{J} / \mathbf{g})$ & $\mathbf{T}_{\mathbf{c}}\left({ }^{\mathbf{0}} \mathbf{C}\right)$ & $\mathbf{X}_{\mathbf{c}}(\mathbf{\%})$ \\
\hline $\mathrm{vPP}$ & 166.8 & 74.6 & 113.1 & 43.3 \\
\hline $\mathrm{rPP}$ & 166.7 & 61.3 & 122.3 & 31.8 \\
\hline A & 164.1 & 24.6 & 122.0 & 14.1 \\
\hline B & 164.5 & 34.4 & 122.5 & 20.2 \\
\hline C & 164.2 & 34.1 & 123.1 & 12.5 \\
\hline D & 163.8 & 28.4 & 122.6 & 18.2 \\
\hline E & 164.2 & 18.9 & 123.0 & 12.2 \\
\hline F & 164.1 & 25.3 & 122.4 & 15.9 \\
\hline G & 164.5 & 17.5 & 122.5 & 11.9 \\
\hline H & 164.2 & 26.5 & 122.1 & 14.3 \\
\hline
\end{tabular}

Es posible apreciar que $\mathrm{PP}$ virgen y $\mathrm{rPP}$ muestran un valor similar para la temperatura de fusión $\mathrm{T}_{\mathrm{m}}$ de $166^{\circ} \mathrm{C}$, lo cual sugiere que el proceso de reciclaje no produce una degradación significativa en el peso molecular del polímero.

La incorporación de 50 y $60 \%$ de harina de madera en $\mathrm{rPP}$ reduce ligeramente el valor de $\mathrm{T}_{\mathrm{m}}$ a $164^{\circ} \mathrm{C}$.

Algunos estudios muestran que otros rellenos para polímeros tampoco producen cambios en las temperaturas de fusión de los compositos. Abu-Sharkh y Hamid (2004), reforzaron PP virgen con 30\% de partículas de hojas de palma, Brachet et al. (2008) utilizaron 10 y $20 \%$ de $\mathrm{CaCO}_{3}$ para reforzar rPP y no se observaron cambios significativos en $\mathrm{T}_{\mathrm{m}}$.

En la tabla 3 se observa un cambio del valor de $\mathrm{T}_{c}$ de $113^{\circ} \mathrm{C}$ para PP virgen a valores sobre $122^{\circ} \mathrm{C}$ para $\mathrm{rPP}$ y sus compositos.

Una posible explicación para este fenómeno es que la presencia de 50 o 60\% de material lignocelulósico en la matriz polimérica reduce el volumen libre de las moléculas del polímero y éste cristaliza a una temperatura mayor cuando es enfriado desde el material fundido. Esto indica que los aditivos que debe contener el rPP y la harina de madera en los compositos actúan como agentes nucleantes que aceleran la velocidad de cristalización.

Una situación similar es reportada en un estudio de cristalización de HDPE reforzado con harina de madera de teca (Sui et al. 2009).

La tabla 3 también muestra una fuerte disminución del porcentaje de cristalinidad, $\mathrm{X}_{\mathrm{c}}$, de $\mathrm{rPP}(30 \%)$ comparado con PP virgen (43\%). La adición de 50\% de harina de madera reduce aún más la cristalinidad a valores entre 16 y $20 \%$ y a valores entre 13 y $16 \%$ cuando el porcentaje de harina de madera es de $60 \%$.

La incorporación de MAPP (muestras A, C, E y G) reduce la cristalinidad a valores entre 10 y 14\% debido a un incremento de la interacción entre las fases de madera y la matriz polimérica.

La tabla 3 muestra que la disminución de la cristalinidad produce una disminución de $\Delta \mathrm{H}_{\mathrm{m}}$ debido a que la entalpía de fusión es proporcional a la cristalinidad.

Otros estudios realizados con PP virgen reforzado con materiales lignocelulósicos presentan resultados diferentes. Cuando se agregan 10 y $20 \%$ de fibra de sisal a PP virgen, aumenta el grado de cristalinidad (X $\mathrm{X}_{\mathrm{c}}$, pero porcentajes superiores al 20\% disminuyen los valores de $\mathrm{X}_{\mathrm{c}}$ (Xie et al. 2002). 
La tabla 4 muestra los resultados de los análisis termogravimétricos de PP virgen, rPP y compositos madera-rPP.

Tabla 4. Propiedades termogravimétricas de PP, rPP y compositos harina de madera-rPP.

\begin{tabular}{|c|c|c|c|}
\hline Muestras & $\mathbf{T}_{\mathbf{5 \%}}\left({ }^{\mathbf{0}} \mathbf{C}\right)$ & $\mathbf{D T G}_{\mathbf{W F}}$ & $\mathbf{D T G}_{\mathbf{P P}}$ \\
\hline vPP & 305 & - & 408 \\
\hline rPP & 350 & - & 445 \\
\hline A & 245 & 354 & 448 \\
\hline B & 248 & 354 & 450 \\
\hline C & 244 & 352 & 446 \\
\hline D & 244 & 350 & 448 \\
\hline E & 259 & 354 & 458 \\
\hline F & 248 & 351 & 455 \\
\hline G & 256 & 352 & 454 \\
\hline H & 254 & 352 & 456 \\
\hline
\end{tabular}

En la tabla 4 se aprecia que la descomposición de PP virgen y de rPP ocurre en una sola etapa que se inicia a $305^{\circ} \mathrm{C}$ en PP virgen y alcanza la máxima velocidad de pérdida de masa (derivada termogravimétrica, DTG) a $408^{\circ} \mathrm{C}$.

En las muestras de rPP, se observa una estabilidad térmica levemente superior iniciándose la descomposición a $350^{\circ} \mathrm{C}$ con un DTG de $445^{\circ} \mathrm{C}$.

Los perfiles de descomposición de los compositos de madera-rPP están caracterizados por dos etapas. La primera descomposición se inicia a $245^{\circ} \mathrm{C}$ y corresponde a la descomposición de los componentes de la madera (celulosa, hemicelulosa y lignina). El DTG para la madera $\left(\mathrm{DTG}_{\mathrm{WF}}\right)$ en los compositos se sitúa en torno a los $350^{\circ} \mathrm{C}$.

La segunda etapa de descomposición corresponde a la degradación de rPP donde los valores promedio de DTG de los compositos con $50 \%$ de harina de madera son de $448^{\circ} \mathrm{C}$ y de $455^{\circ} \mathrm{C}$ para los compositos con $60 \%$ de harina de madera.

El incremento del porcentaje de harina de madera de 50 a 60\% (para 60 y 100 mesh) aumenta ligeramente la estabilidad térmica de los compositos iniciándose la descomposición de rPP a temperaturas de unos $10^{\circ} \mathrm{C}$ más alta.

El incremento de la estabilidad térmica de rPP y de los compositos de madera-rPP, comparados con PP virgen puede ser causada por aditivos incorporados en la fabricación de los sacos de rafia y de que el proceso de reciclaje no produjo una degradación significativa del peso molecular del polímero.

Otros estudios realizados con harina de madera como refuerzo de una mezcla de PP y LDPE muestran también un ligero aumento de la estabilidad térmica de los polímeros con la incorporación de la madera en la matriz polimérica (Dikobe y Luyt 2010). 


\section{CONCLUSIONES}

En el proceso de mezclado se aprecia un fuerte incremento del torque al adicionar la madera, pero después de 10 minutos de proceso, los valores del torque de PP virgen, rPP y compositos madera-rPP no presentan diferencias apreciables. Cuando el contenido de harina de madera fue de 50\% los valores promedio del torque del proceso fueron de $9 \mathrm{Nm}$. Al aumentar el contenido de madera a $60 \%$, este valor aumentó a 10Nm. El tamaño de partículas no produjo diferencias apreciables en el torque.

El MOR, determinado en ensayos de tracción de los compositos madera-rPP, disminuye respecto de los polímeros puros. Los valores del MOE en ensayos de tracción y flexión de los compositos madera-rPP, fueron superiores a los polímeros puros, lo cual evidencia un aumento de la rigidez de las mezclas, respecto de los polímeros puros.

La resistencia al impacto de rPP y de los compositos madera-rPP fueron notoriamente superiores al PP virgen.

La variación del porcentaje de madera de 50 a $60 \%$ no muestra un efecto claro en las propiedades mecánicas del material y el tamaño de partícula no influye significativamente en estas propiedades.

En general, los valores más altos del MOR, MOE y resistencia al impacto de los compositos madera-rPP fueron obtenidos cuando se utilizó MAPP como agente acoplante, lo cual indica un incremento de la interacción en la interfase madera-polímero en estas muestras.

Esta variación en las propiedades mecánicas son concordantes con los resultados de ensayos de las propiedades térmicas que muestran una fuerte disminución del grado de cristalinidad de PP virgen y de rPP cuando se incorpora harina de madera en la matriz polimérica.

El análisis térmico mostró que la presencia de madera en rPP produce un leve aumento de la estabilidad térmica del polímero. En general, es posible concluir que es factible fabricar materiales compuestos con buenas propiedades térmicas y mecánicas utilizando PP postconsumo reforzado con 50 o $60 \%$ de harina de madera con tamaños de partículas de 60 o 100 mesh.

\section{AGRADECIMIENTOS}

Los autores agradecen el apoyo financiero de CONICYT REGIONAL/CIPA R08C1002 


\section{BIBLIOGRAFÍA}

Abu-Sharkh, B.F.; Hamid, H. 2004. Degradation study of date palm fibre/ polypropylene composites in natural and artificial weathering: mechanical and thermal analysis. Polymer Degradation and Stability 85(3): 967-973.

Adhikary, K.B.; Pang, S.; Staiger, M.P. 2010. Effects of the accelerated freeze-thaw cycling on physical and mechanical properties of wood flour-recycled thermoplastic composites. Polymer Composites 31(2): 185-194.

Al-Salem, S.M.; Lettieri, P.; Baeyens, J. 2009. Recycling and recovery routes of plastic solid waste (PSW): A review. Waste Management 29(10): 2625-2643.

Ashori, A.; Nourbakhsh, A. 2009. Characteristics of wood-fiber plastic composites made of recycled materials. Waste Management 29(4): 1291-1295.

ASTM. American Standard Test Methods. 2004. Standard Test Method for Melt Flow Rates of Thermoplastics by Extrusion Plastometer.D 1238-04. ASTM International, West Conshohocken, PA.

ASTM. American Standard Test Methods. 2004. Standard Test Methods for Evaluating Properties of Wood -Base Fiber and Particle Panel Materials. D 1037-96a ASTM International, West Conshohocken, PA.

ASTM.American Standard Test Methods. 2004. Standard Test Methods for Flexural Properties of Unreinforced and Reinforced Plastics and Electrical Insulating Materials. D 790-03. ASTM International, West Conshohocken, PA.

ASTM. American Standard Test Methods. 2004. Standard Test Methods for Tensile Properties of Plastics. D 638-03. ASTM International, West Conshohocken, PA.

ASTM. American Standard Test Methods. 2004. Standard Test Methods for Determining the Izod Pendulum Impact Resistance of Plastics. D 256-03. ASTM International, West Conshohocken, PA.

Borysiak, S.; Doczealska, B. 2006. Influence of chemical modification of wood on the crystallization of polypropylene. Holz als Roh-und Werkstoff 64(6): 451-454.

Bouza, R.; Lasagabaster, A.; Abad, M.J.; Barral, L. 2008. Effects of vinyltrimethoxy silane on thermal properties and dynamic mechanical properties of polypropylene-wood flour composites. Journal of Applied Polymer Science 109(2): 1197-1204.

Brachet, P.; Hoydal, L.T.; Hinrichsen, E.L.; Melum, F. 2008. Modification of mechanical properties of recycled polypropylene from post-consumer containers. Waste Management 28(12): 2456-2464.

Choi, N.W.; Mori, I.; Ohama, Y. 2006. Development of rice husks-plastic composites for building materials. Waste Management 26(2): 189-194.

Clemons, C.M.; Caufield, D.F. 2005. Natural fibers. In Functional Fillers for plastics. Wiley, Weinheim.

Dányádi, L. ; Moczó, J. ; Pukánszky, B. 2010. Effect of various surface modifications of wood flour on the properties of PP/wood composites. Composites: Part A 41(2): 199-206.

Dikobe, D.G.; Luyt, A.S. 2010. Comparative study of the morphology and properties of PP/LLDPE/wood powder and MAPP/LLDPE/wood powder polymer blend composites. Express Polymer Letters 4(11): 729-741.

Galeski, A. 1999. Crystallization. In Polypropylene: An A-Z reference. Kluwer, Dordrecht. 
Haque, M.M.; Hasan, M.; Islam, MS.; Ali, ME. 2009. Physico-mechanical properties of chemically treated palm and coir fiber reinforced polypropylene composites. Bioresource Technology 100(20): 4903-4906.

Keener, T.J.; Stuart, R.K.; Brown, T.K. 2004. Maleated coupling agents for natural fibre composites. Composites Part A : Applied Science and Manufacturing 35(3): 357-362.

Lisperguer, J.; Bustos, X.; Saravia, Y. 2011. Thermal and mechanical properties of wood flour-polystyrene blends from postconsumer plastic waste. Journal of Applied Polymer Science 119(1): 443-451.

Najafi, S.K.; Hamidinia, E.; Tajvidi, M. 2006. Mechanical properties of composites from sawdust and recycled plastics. Journal of Applied Polymer Science 100(5): 3541-3645.

Nuñez, A.J.; Sturm, P.C.; Kenny, J.M.; Aranguren, M.I.; Marcovich, N.E.; Reboredo, M.M. 2003. Mechanical characterization of polypropylene-wood flour composites. Journal of Applied Polymer Science 88(9): 1420-1428.

Salemane, M. G.; Luyt, A.S. 2006. Thermal and mechanical properties of polypropylene-wood powder composites. Journal of Applied Polymer Science 100(5): 4173-4180.

Selke, S.E.; Wichman, I. 2004. Wood fiber / polyolefin composites. Composites Part A : Applied Science and Manufacturing 35(3): 321-326.

Simonsen, J.; Rials, T.G. 1996. Morphology and properties of wood-fiber reinforced blends of recycled polystyrene and polyethylene. Journal of Thermoplastic Composite Materials 9(3): 292-302.

Spinacé, M.A.; Fermoseli, K.K.G.; De Paoli, M.A. 2009. Recycled polypropylene reinforced with Curaua fibers by extrusion. Journal of Applied Polymer Science 112(6): 3686-3694.

Sui, G.; Fuqua, M.A.; Ulven, C.A.; Zhong, W.H. 2009. A plant fiber reinforced polymer composite prepared by twin-screw extruder. Bioresource Technology 100(3): 1246-1251.

Xie, X.L.; Li, R.K.Y.; Tjong, S.C.; Mai, Y.W. 2002. Structural properties and mechanical behavior of injection molded composites of polypropylene and sisal fiber. Polymer Composites 23(3): 319-328.

Xiong, C.; Qi, R.; Wang, Y. 2009. Wood-thermoplastic composites from wood flour and high-density polyethylene. Journal of Applied Polymer Science 114(2): 1160-1168.

Yeh, S.; Kim, K.; Gupta, R.K. 2013. Synergistic effect of coupling agents on polypropylene-based woodplastic composites. Journal of Applied Polymer Science 127(2): 1047-1053. 